\title{
Estrutura da comunidade de Phlebotominae (Diptera, Psychodidae) em mata ciliar do município de Urbano Santos, Maranhão, Brasil
}

\author{
Fredgardson C. Martins ${ }^{1}$, Jorge L. P. Moraes ${ }^{2}$, Nivaldo Figueiredo ${ }^{3}$ \& José M. M. Rebêlo ${ }^{2,3}$ \\ 1. Centro de Ciências Agrárias e Ambientais, Universidade Federal do Maranhão, BR 222, km 04, 65500-000 Chapadinha, MA, Brasil. \\ (fred@ufma.br) \\ 2. Laboratório de Entomologia e Vetores, Universidade Federal do Maranhão, Praça Madre Deus n 0 02, 65025-560 São Luís, MA, Brasil. \\ 3. Departamento de Biologia, Universidade Federal do Maranhão, Avenida dos Portugueses s/n, Campus do Bacanga, São Luís, MA, Brasil. \\ (macariorebelo@uol.com.br)
}

\begin{abstract}
Structure of the Phlebotominae (Diptera, Psychodidae) community in a gallery forest in Urbano Santos district, state of Maranhão, Brazil. The diversity, relative abundance and distribution of Phlebotominae was examined in three sectors (edges and center) of a fragment of gallery forest in the municipality of Urbano Santos, Maranhão, Brazil. Specimens were captured in June and November, 2003, and in January and March, 2004, between 06:00 PM and 06:00 AM. At each collection night, 18 light traps were installed, six in each forest sector, totaling 864 hours of work. Seventeen species were found. The center of the fragment had the greatest diversity of species (14), followed by edge B (13) and edge A (12). The species Lutzomyia infraspinosa (Mangabeira, 1941), L. flaviscutellata (Mangabeira, 1942) and L. evandroi (Costa Lima \& Antunes, 1936) were the only ones that appeared as dominants in all forest sectors. Fourteen species were found in both seasons; L. fluviatilis (Floch \& Abonnenc, 1944) was found only in the rainy season (January and March); and L. migonei (França, 1920) and L. pinottii (Damasceno \& Arouck, 1956) in the dry season (June and November). The differences regarding the abundance of individuals between the seasons were not statistically significant. The frequent presence of L. flaviscutellata may explain a case of diffuse cutaneous leishmaniasis in a patient of that municipality.
\end{abstract}

KEYWORDS. Vector ecology, Lutzomyia, Cerrado vegetation.

RESUMO. A diversidade, abundância relativa e a distribuição de Phlebotominae foram estudadas em três setores (bordas e centro) de um fragmento de mata ciliar no município de Urbano Santos, Maranhão, Brasil. Os espécimes foram capturados em junho e novembro/2003 e em janeiro e março/2004 das 18 às 6 horas. Em cada noite de coleta foram instaladas 18 armadilhas, seis em cada setor da mata, totalizando um esforço de 864 horas. Foram encontradas 17 espécies. O centro do fragmento obteve a maior riqueza de espécies (14), seguido da borda B (13) e da borda A (12). As espécies Lutzomyia infraspinosa (Mangabeira, 1941), L. flaviscutellata (Mangabeira, 1942) e L. evandroi (Costa Lima \& Antunes, 1936) foram as únicas que apareceram como dominantes nos três setores da mata. Quatorze espécies ocorreram em ambas estações, sendo que L. fluviatilis (Floch \& Abonnenc, 1944) foi encontrada apenas na estação chuvosa (janeiro e março) e L. migonei (França, 1920) e L. pinottii (Damasceno \& Arouck, 1956) apenas na estação seca (junho e novembro). As diferenças registradas na abundância de indivíduos entre as estações não foram estatisticamente significativas. A presença frequente de $L$. flaviscutellata pode explicar um caso de leishmaniose cutânea difusa em uma paciente deste município.

PALAVRAS-CHAVE. Ecologia de vetor, Lutzomyia, vegetação de Cerrado.

O Nordeste do Maranhão destaca-se por situar-se numa zona de transição no tocante à composição da vegetação e no tipo de clima. Assim, observam-se zonas de ocorrência de floresta estacional perenifólia aberta associada ora com babaçu, ora com cerrado/caatinga, ou com ambos, subordinadas a um clima semi-úmido e/ou semi-árido (IBGE, 1984). Dentro dessas zonas podem ser encontrados outros tipos de formação, como a mata ciliar. Essa transição vegetacional e climática afeta a distribuição dos flebotomíneos (REBÊLo et al., 1996), sendo possível encontrar nessa região espécies Amazônicas (REBÊLo et al., 2000a, b) e outras frequentes nas áreas nordestinas (REBÊLo et al., 1999).

Em função das características mencionadas, há superposição das duas formas de leishmanioses, a cutânea que, de modo geral, é mais evidente nas áreas amazônicas e a visceral (calazar), muito comum no lado nordestino (Costa et al., 2005).

$\mathrm{O}$ intenso fluxo migratório e o incessante processo de modificação da cobertura vegetal que ocorre em praticamente todo o Estado vêm contribuindo para o estabelecimento dessa nova realidade da epidemiologia das leishmanioses (MARTINs et al., 2004).

A região deste estudo não difere da realidade do Estado, apresentando um intenso processo de supressão e/ou substituição das formações florestais naturais, o que tem provocado visíveis alterações na paisagem, sendo poucos e geralmente pequenos os remanescentes naturais destas formações (FIGUEIREDO \& ANDRADE, 2007).

A redução das formações florestais naturais pela introdução de culturas agrícolas, reflorestamento, pecuária e expansão urbana tem afetado a diversidade, a densidade e as estratégias de sobrevivência de inúmeros grupos de plantas e animais, inclusive de flebotomíneos, pois esse processo vem selecionando espécies com capacidade de colonizar as áreas modificadas e até mesmo o ambiente peridomiciliar (MAYRINK et al., 1979; AZEVEDO et al., 1990).

No presente trabalho estudou-se a distribuição de flebotomíneos em três diferentes setores de um fragmento de Mata Ciliar de um dos principais rios de Urbano Santos, Maranhão, Brasil, um município submetido ao estresse pelo desmatamento, reflorestamento e outras atividades antrópicas. 


\section{MATERIAL E MÉTODOS}

$\mathrm{O}$ estudo foi realizado na propriedade da fazenda Santo Amaro, município de Urbano Santos, Maranhão,

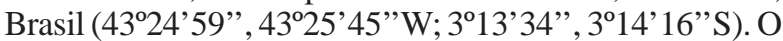
local situa-se na zona fisiográfica do Baixo Parnaíba, distando aproximadamente $5 \mathrm{~km}$ da sede do município, cuja altitude é de $41 \mathrm{~m}$.

A área total da fazenda é de 123,122 ha, dos quais 26,922 ha são plantados com eucalipto de diferentes variedades e sub-bosque composto por espécies do Cerrado e das matas adjacentes. A maior parte da área é ocupada por duas formações florestais distintas: um fragmento de mata ciliar do rio Mocambo (um dos principais rios do município) e um fragmento de Mata Mesófila Semidecídua, imediatamente após a mata ciliar.

O estudo teve como foco o fragmento de mata ciliar que se estende pela margem direita do rio Mocambo (sentido montante-jusante). O limite norte se dá com uma estreita área aberta em moderado nível de regeneração, imediatamente seguido por uma plantação de eucalipto com aproximadamente 20 anos de idade. No limite oeste está a Mata Mesófila Semidecídua, constituindo-se quase como um prolongamento da mata ciliar. Em alguns trechos é praticamente impossível a distinção entre essas formações. No limite sul encontra-se o riacho Santo Amaro, um afluente do rio Mocambo.

As áreas mais próximas do rio permanecem alagadas durante boa parte da estação chuvosa (dezembro a maio), enquanto algumas áreas intermediárias só alagam após as fortes chuvas (mesmo no período chuvoso), permanecendo assim por alguns dias, dependendo do volume precipitado. As áreas mais elevadas (mais próximas da Mata Mesófila) não sofrem alagamento sazonal.

Para definição dos pontos de amostragem, o fragmento de mata ciliar foi dividido em três setores (borda A, centro e borda B). Para delimitação desses setores foram instalados três transectos paralelos à margem do rio. A distância entre os transectos foi estabelecida ao acaso. Em cada um dos três transectos foram alocados, a cada $15 \mathrm{~m}$, um ponto. A borda A (setor norte do fragmento) correspondeu aos primeiros trinta pontos de cada um dos transectos, sendo os dois primeiros utilizados para amostragem dos flebotomíneos. O centro (setor central do fragmento) corresponde aos trinta pontos centrais de cada transecto, sendo os dois pontos centrais utilizados para amostragem dos flebotomíneos. A borda B (setor sul do fragmento) corresponde aos trinta últimos pontos de cada um dos transectos, sendo os dois últimos utilizados para amostragem dos flebotomíneos.

Desse modo, em cada setor do fragmento foi instalado um conjunto de 6 armadilhas luminosas modelo CDC movidas à pilha posicionadas a uma altura de $1,5 \mathrm{~m}$, sempre nos mesmos locais. A distância entre as armadilhas de um mesmo transecto foi de $15 \mathrm{~m}$ e entre o conjunto de armadilhas foi de aproximadamente $780 \mathrm{~m}$. As capturas foram realizadas em junho e novembro de 2003 (estiagem) e em janeiro e março de 2004 (estação chuvosa), sempre em noites com baixa claridade para não interferir na eficiência das armadilhas. As 18 armadilhas funcionaram por $12 \mathrm{~h}$ consecutivas em cada coleta. O esforço de campo totalizou $864 \mathrm{~h}$.
Todos os espécimes capturados foram sacrificados em câmara refrigerada e identificados no Laboratório de Entomologia e Vetores da Universidade Federal do Maranhão por técnicos da UFMA/FUNASA auxiliados pela chave de identificação proposta por Young \& DUNCAN (1994).

Análise dos dados. Utilizou-se o teste Quiquadrado $(\alpha=0,05)$ para analisar as diferenças na proporção entre machos e fêmeas capturados. A análise de variância fatorial $(2 \times 3)$ foi utilizada para avaliar as diferenças nos números de espécimes capturados entre os setores da mata e as duas estações. As armadilhas que não obtiveram sucesso na captura dos flebotomíneos foram excluídas das análises estatísticas.

Para a análise de diversidade utilizou-se o índice de Shannon-Wienner (H') (PIELou, 1975) em função do grande número de espécies representadas por poucos indivíduos (KREBS, 1989). A equitabilidade foi calculada pelo índice de Pielou (J') e a similaridade da composição de espécies entre os setores do fragmento foram calculadas pelos índices de Jaccard (CCj, qualitativo) e Morisita-Horn ( $\mathrm{C}_{\mathrm{H}}$, quantitativo) (KREBS, 1989).

Para a análise da abundância relativa (\%) e seus limites de confiança foi utilizado o método de KaTo et al. (1952). As espécies foram consideradas dominantes quando seu limite de confiança inferior fosse maior que o limite superior para espécies ausentes.

\section{RESULTADOS}

Foram encontradas 17 espécies de Lutzomyia França, 1924: L. damascenoi (Mangabeira, 1941), L. evandroi (Costa Lima \& Antunes, 1936), L. flaviscutellata (Mangabeira, 1942), L. fluviatilis (Floch \& Abonnenc, 1944), L. infraspinosa (Mangabeira, 1941), L. lenti (Mangabeira, 1938), L. longipalpis (Lutz \& Neiva, 1912), L. longipennis (Barreto, 1946), L. migonei (França, 1920), L. monstruosa (Floch \& Abonnenc, 1944), L. pinottii (Damasceno \& Arouk, 1956), L. richardwardi Ready \& Fraiha, 1981, L. sordellii (Shannon \& Del Pont, 1927), L. termitophila Martins, Falcão \& Silva, 1971, L. trinidadensis (Newstead, 1922), L. whitmani (Antunes \& Coutinho, 1939) e L. wellcomei (Fraiha, Shaw \& Lainson, 1971).

A diversidade de flebotomíneos para o fragmento foi de $\mathrm{H}^{\prime}=2,015$ nats/indivíduos e a equabilidade foi de 0,711 (Tab. I).

Foram capturados 306 espécimes (47,39\% machos e 52,61\% fêmeas) (Tab. II). Não houve diferença estatística significativa entre essas proporções $\left(\chi^{2}\right.$-Yates $=0,837$; $\mathrm{p}=0,3912)$. As espécies mais abundantes foram $L$. infraspinosa (41,18\%), L. flaviscutellata $(17,32 \%), L$. evandroi $(9,15 \%)$, L. monstruosa $(5,56 \%)$, L. termitophila $(4,58 \%)$, L. sordellii $(4,25 \%)$, L. trinidadensis $(3,92 \%)$, L.whitmani $(3,27 \%)$, L. lenti $(2,61 \%)$, L. wellcomei $(2,29 \%), L$. longipalpis $(1,63 \%)$ e $L$. aff. longipennis $(1,63 \%)$ e seus limites de confiança $(p=0,05)$ (intervalos de confiança, ver Fig. 1). As demais 5 espécies representaram juntas $2,61 \%$.

A riqueza de espécies foi maior no centro (14), seguido das bordas B (13) e A (12). A abundância de indivíduos entre os setores não apresentou diferenças 
significativas ( $F=0,8537$; gl. 2, 57; $p=0,5656)$ (Tab. I). A diversidade também foi maior no centro $\left(H^{\prime}=2,14\right)$, seguido da borda $A\left(H^{\prime}=1,91\right)$ e da borda $B\left(H^{\prime}=1,76\right)$ (Tab. I). A equitabilidade também foi maior no centro do fragmento $\left(\mathrm{J}^{\prime}=0,8111\right)$ e menor na borda $\mathrm{B}\left(\mathrm{J}^{\prime}=0,6865\right)$ (Tab. I).

Utilizando dados quantitativos, a similaridade foi maior entre a borda $A$ e o centro $\left(\mathrm{C}_{\mathrm{H}}=0,93\right)$, entre as bordas $\left(\mathrm{C}_{\mathrm{H}}=0,8931\right)$ e menor entre o centro e a borda $\mathrm{B}$ $\left(\mathrm{C}_{\mathrm{H}}=0,8642\right)$. Entretanto, quando utilizados dados binários, a maior similaridade foi entre a borda $\mathrm{B}$ e o centro ( $\mathrm{CCj}=0,80,12$ espécies em comum), entre bordas $(\mathrm{CC}=0,667,10$ espécies em comum) e entre a borda A e o centro $(\mathrm{CCj}=0,625,10$ espécies em comum), respectivamente. Em relação às espécies exclusivas de cada setor, a borda A apresentou 2 espécies exclusivas, $\mathrm{o}$ centro 2 espécies e a borda $\mathrm{B}$ apenas uma espécie.

A abundância dos flebotomíneos não foi diferente entre os setores $(F=0,5812 ; \mathrm{gl} .2,69 ; \mathrm{p}=0,5671)$, nem mesmo quando consideradas as diferentes estações independentemente (estiagem: $\mathrm{F}=0,4136$; gl. 2,$33 ; \mathrm{p}=$ 0,67 e estação chuvosa: $\mathrm{F}=0,7086 ; \mathrm{gl} .2,33 ; \mathrm{p}=0,5039$ ).

As espécies dominantes, com exceção de $L$. infraspinosa, variaram de acordo com o setor do fragmento. Na borda A foram: L. infraspinosa $(47,1 \%)$, L. evandroi (15,3\%), L. flaviscutellata $(10,6 \%)$ e L. sordellii $(5,9 \%)$ (Fig. 2). No centro: L. infraspinosa $(37,4 \%)$, L. sordellii $(9,1 \%)$, L. trinidadensis $(9,1 \%)$, L. flaviscutellata $(8,1 \%)$,

Tabela I. Estrutura da comunidade de flebotomíneos entre os setores (bordas e centro) e para o fragmento de mata ciliar do rio Mocambo, Município de Urbanos Santos, Maranhão, Brasil (2003-2004).

\begin{tabular}{|c|c|c|c|c|}
\hline Espécies & Borda A & Centro & Borda B & Total \\
\hline Lutzomyia infraspinosa & 40 & 37 & 49 & 126 \\
\hline L. flaviscutellata & 9 & 8 & 36 & 53 \\
\hline L. evandroi & 13 & 9 & 6 & 28 \\
\hline L. monstruosa & 4 & 7 & 6 & 17 \\
\hline L. termitophila & - & 8 & 6 & 14 \\
\hline L. sordellii & 5 & 3 & 5 & 13 \\
\hline L. trinidadensis & 2 & 9 & 1 & 12 \\
\hline L. whitmani & 4 & 3 & 3 & 10 \\
\hline L. lenti & 1 & 4 & 3 & 8 \\
\hline L. wellcomei & 2 & 2 & 3 & 7 \\
\hline L. longipalpis & 1 & 2 & 2 & 5 \\
\hline L. aff. longipenis & & 5 & & 5 \\
\hline L. damascenoi & & 1 & 1 & 2 \\
\hline L. fluviatalis & 2 & & & 2 \\
\hline L. richardwardi & 2 & & & 2 \\
\hline L. migonei & & & 1 & 1 \\
\hline L. pinottii & & 1 & & 1 \\
\hline Números de espécimes & 85 & 99 & 122 & 306 \\
\hline Números de espécies & 12 & 14 & 13 & 17 \\
\hline Índice de Diversidade de Shannon (H') & 1,9140 & 2,1405 & 1,7600 & 2,0147 \\
\hline Equitabilidade $\left(\mathrm{J}^{\prime}\right)$ & 0,7209 & 0,8111 & 0,6865 & 0,7111 \\
\hline
\end{tabular}

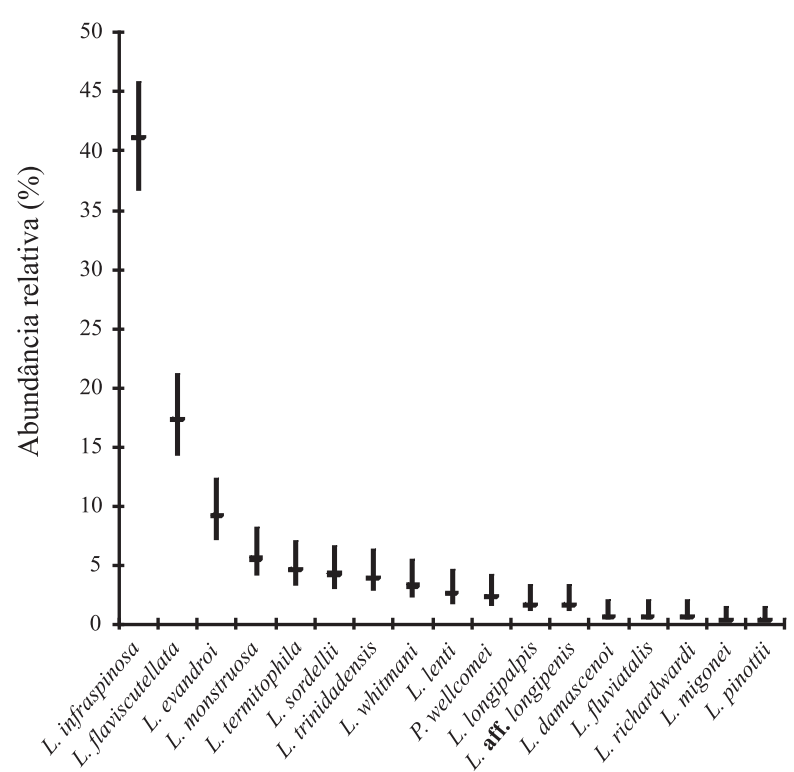

Espécies

Figura 1. Abundância relativa (\%) e seus limites de confiança dos flebotomíneos da mata ciliar do rio Mocambo, município de Urbano Santos, Maranhão, Brasil (2003-2004).

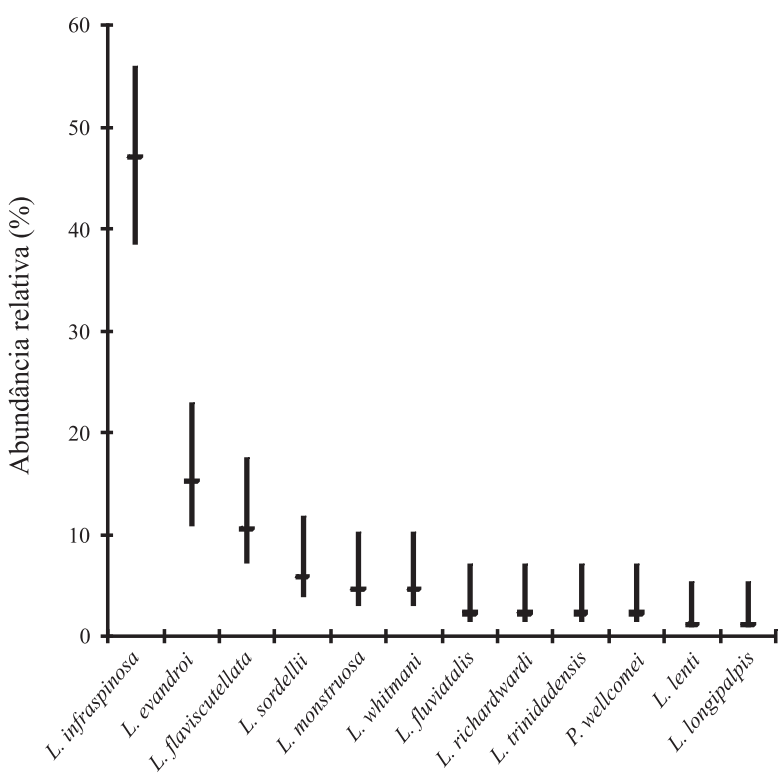

Espécies

Figura 2. Abundância relativa (\%) e seus limites de confiança dos flebotomíneos na borda A do fragmento de mata ciliar do rio Mocambo, município de Urbano Santos, Maranhão, Brasil (2003-2004). 
L. termitophila $(8,1 \%)$, L. monstruosa $(7,1 \%)$ e $L$. aff. longipennis (5\%) (Fig. 3). Na borda B: L. infraspinosa $(40,2 \%)$, L. flaviscutellata $(29,5 \%)$, L. evandroi $(4,9 \%)$, L. monstruosa $(4,9 \%)$, L. termitophila $(4,9 \%)$ e L. sordellii $(4,1 \%)$ (Fig. 4).

Quatorze espécies estiveram presentes nas duas estações, L. fluviatilis só foi encontrada na estação chuvosa e L. migonei e L. pinottii na estiagem, sendo que estas espécies têm ocorrência ocasional. As diferenças na abundância de indivíduos entre as estações não foram significativas, apesar de apresentarem uma tendência de ser maior no período de estiagem $(56,2 \%$ na estiagem e $43,8 \%$ no período chuvoso) $(\mathrm{F}=1,5435 ; \mathrm{gl} .1,58 ; \mathrm{p}=0,2168)$. Entre as espécies dominantes, somente L. flaviscutellata e L. evandroi foram estatisticamente mais abundantes na estiagem
$(\mathrm{F}=5,097$; gl. 1,$70 ; \mathrm{p}=0,0255$ e $\mathrm{F}=8,6517$; gl. 1,70 ; $\mathrm{p}=0,0047$, respectivamente) (Tab. II).

Foram capturados 80 machos e 92 fêmeas no período da estiagem e 65 machos e 69 fêmeas na estação chuvosa (Tab. II). Essas diferenças nas proporções entre machos e fêmeas não foram significativas em nenhuma das estações (estiagem: $\chi^{2}$-Yates $=0,703 ; p=0,4016$; estação chuvosa: $\chi^{2}$-Yates $=0,067 ; \mathrm{p}=0,7955$ ).

$\mathrm{Na}$ estiagem, as espécies dominantes foram: $L$. infraspinosa (30,81\%), L. flaviscutellata $(27,32 \%), L$. evandroi $(14,53 \%)$, L. trinidadensis $(4,65 \%)$, L. lenti $(4,07 \%)$, L. monstruosa $(2,91 \%)$ e L. whitmani $(2,91 \%)$. Na estação chuvosa as dominantes foram: $L$. infraspinosa (54,48\%), L. monstruosa (8,95\%), L. sordellii $(7,46 \%), L$. termitophila $(7,46 \%)$, L. flaviscutellata $(4,48 \%)$ e $L$. whitmani $(3,73 \%)$ (Tab. II).

Tabela II. Flebotomíneos capturados por estação do ano, no fragmento de mata ciliar do do rio Mocambo, município de Urbanos Santos, Maranhão, Brasil ( $\chi^{2}$, Qui-quadrado de Yates; M, machos; F, fêmeas; T, total).

\begin{tabular}{|c|c|c|c|c|c|c|c|c|c|c|}
\hline \multirow{2}{*}{$\begin{array}{l}\text { Estações } \\
\text { Espécies }\end{array}$} & \multicolumn{3}{|c|}{$\begin{array}{c}\text { Estiagem } \\
\chi^{2}=0,703 ; \mathrm{p}=0,4016\end{array}$} & \multicolumn{3}{|c|}{$\begin{array}{c}\text { Chuvosa } \\
\chi^{2}=0,067 ; p=0,7955\end{array}$} & \multicolumn{4}{|c|}{$\begin{array}{c}\text { Total } \\
\chi^{2}=0,837 ; p=0,3912\end{array}$} \\
\hline & $\mathrm{M}$ & $\mathrm{F}$ & $\mathrm{T}$ & $\mathrm{M}$ & $\mathrm{F}$ & $\mathrm{T}$ & M & $\mathrm{F}$ & $\mathrm{n}^{\mathrm{O}}$ & $\%$ \\
\hline Lutzomyia infraspinosa & 26 & 27 & 53 & 39 & 34 & 73 & 65 & 61 & 126 & 41,18 \\
\hline L. flaviscutellata & 27 & 20 & 47 & 4 & 2 & 6 & 31 & 22 & 53 & 17,32 \\
\hline L. evandroi & 3 & 22 & 25 & 1 & 2 & 3 & 4 & 24 & 28 & 9,15 \\
\hline L. monstruosa & & 5 & 5 & 6 & 6 & 12 & 6 & 11 & 17 & 5,56 \\
\hline L. termitophila & & 4 & 4 & 1 & 9 & 10 & 1 & 13 & 14 & 4,58 \\
\hline L. sordellii & 1 & 2 & 3 & 4 & 6 & 10 & 5 & 8 & 13 & 4,25 \\
\hline L. trinidadensis & 7 & 1 & 8 & 2 & 2 & 4 & 9 & 3 & 12 & 3,92 \\
\hline L. whitmani & 1 & 4 & 5 & 3 & 2 & 5 & 4 & 6 & 10 & 3,27 \\
\hline L. lenti & 7 & & 7 & 1 & & 1 & 8 & & 8 & 2,61 \\
\hline L. wellcomei & & 3 & 3 & 1 & 3 & 4 & 1 & 6 & 7 & 2,29 \\
\hline L. longipalpis & 3 & 1 & 4 & 1 & & 1 & 4 & 1 & 5 & 1,63 \\
\hline L. aff. longipenis & 2 & 2 & 4 & & 1 & 1 & 2 & 3 & 5 & 1,63 \\
\hline L. damascenoi & 1 & & 1 & 1 & & 1 & 2 & & 2 & 0,65 \\
\hline L. fluviatalis & & & & & 2 & 2 & & 2 & 2 & 0,65 \\
\hline L. richardwardi & 1 & & 1 & 1 & & 1 & 2 & & 2 & 0,65 \\
\hline L. migonei & & 1 & 1 & & & & & 1 & 1 & 0,33 \\
\hline L. pinottii & 1 & & 1 & & & & 1 & & 1 & 0,33 \\
\hline Total & 80 & 92 & 172 & 65 & 69 & 134 & 145 & 161 & 306 & 100 \\
\hline
\end{tabular}

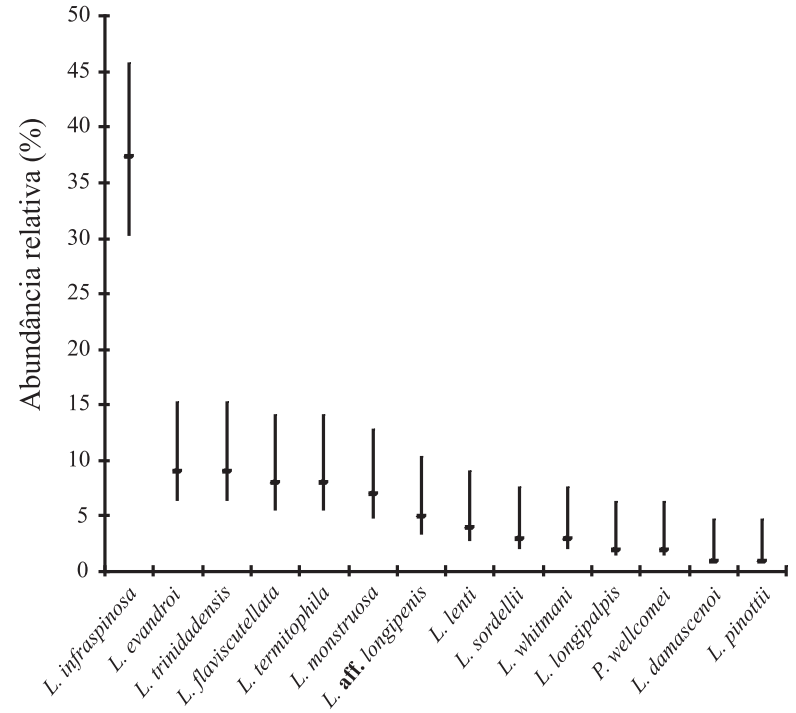

Espécies

Figura 3. Abundância relativa (\%) e seus limites de confiança dos flebotomíneos no centro do fragmento de mata ciliar do rio Mocambo, município de Urbano Santos, Maranhão, Brasil (2003-2004).

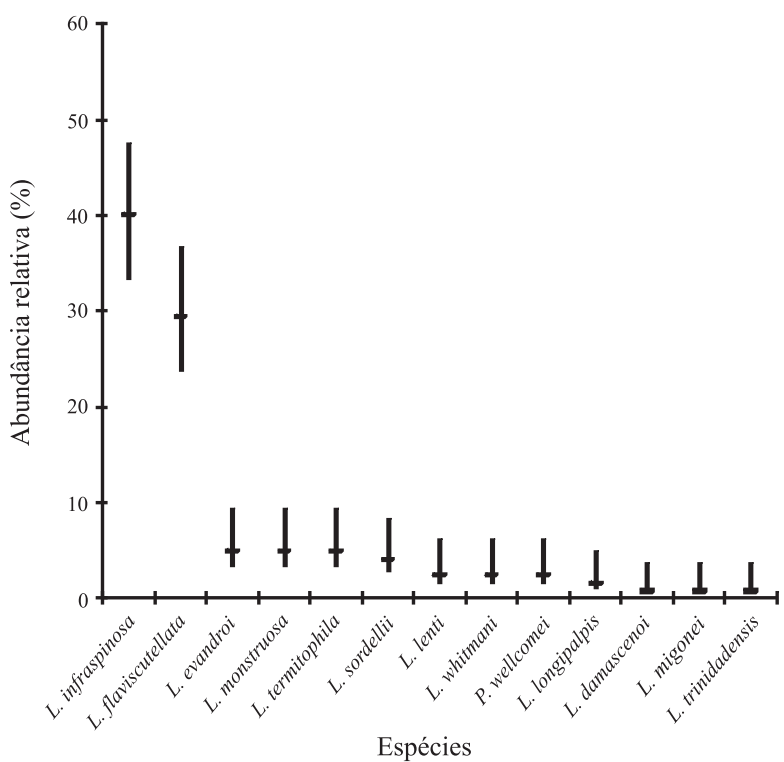

Figura 4. Abundância relativa (\%) e seus limites de confiança dos flebotomíneos na borda B do fragmento de mata ciliar do rio Mocambo, município de Urbano Santos, Maranhão, Brasil (2003-2004). 


\section{DISCUSSÃO}

A comunidade de flebotomíneos encontra-se amplamente distribuída na mata ciliar do município de Urbano Santos. As diferenças entre os setores aparentemente não afetaram de maneira marcada a estrutura da comunidade, embora tenha-se percebido mudança na ordem de dominância para algumas espécies (mas não de L. infraspinosa, a espécie mais frequente da área).

A diversidade neste estudo foi maior do que aquela obtida no cerrado do município de Santa Quitéria (à 200 km), na periferia da sede municipal de Codó (à 300 km) e em Caxias (à $400 \mathrm{~km}$ ), todos situados na mesma zona climática da área deste estudo (REBÊLo et al., 1999; MARTIN \& REBÊLO, 2006).

Entre as espécies encontradas neste estudo, $L$. damascenoi e $L$. pinottii, constituíram o primeiro registro para o Maranhão. Em acréscimo citamos ainda $L$. flaviscutellata, L. fluviatilis, L. infraspinosa, L. migonei, L. monstruosa e L. richardwardi, que juntamente com aquelas encontradas por REBÊLO et al. (1999), perfazem vinte e quatro espécies conhecidas para a região Nordeste do Maranhão.

Apesar da grande riqueza de espécies, a densidade de indivíduos de muitas delas foi muito baixa. Resultados semelhantes foram obtidos por ReBÊLo et al. (2000b) em ambiente florestal da Amazônia maranhense. Essa baixa frequência de indivíduos pode ser resultado da interação de diversos fatores, como a ausência de fontes alimentares sanguíneas, de criadouros e abrigos nas proximidades dos pontos de coleta ou do raio de atração das armadilhas, conforme discutido por MARTIN \& REBÊLO (2006). Ainda assim essas espécies aparentemente raras e, como tal, sem importância na epidemiologia das leishmanioses, podem adaptar-se ao novo meio devido às alterações nas comunidades e desempenhar funções importantes como vetores de Leishmania.

A dominância de L. infraspinosa nesse estudo é curiosa, pois até o momento só havia registro dessa espécie no lado oeste do Estado e, ainda assim, em baixa densidade (REBÊLo et al., 2000a, b). Tal dominância pode indicar a pouca sensibilidade desse flebotomíneo às alterações existentes nos diferentes setores e, nesse sentido, pode vir a se tornar uma das espécies com grande potencial de adaptação a ambientes antropizados. $\mathrm{O}$ mesmo raciocínio pode ser seguido para $L$. flaviscutellata, que ainda não havia sido encontrada em nenhum dos estudos realizados no interior do Maranhão, sendo raros os exemplares capturados na ilha de São Luís. De qualquer forma, a sua predominância pode explicar o caso de leishmaniose cutânea difusa diagnosticado em uma paciente moradora na sede municipal de Urbano Santos (Costa et al., 1992).

Destaca-se ainda a presença de $L$. lenti e $L$. evandroi que têm aparecido em todos os levantamentos realizados nos municípios do nordeste do Estado (REBÊLO et al., 1999; LeONARDo \& Rebêlo, 2004; Martin \& Rebêlo, 2006) e de L. whitmani sempre presente nas áreas silvestres e peridomiciliares rurais (REBÊLo et al. 1999, 2000a, b), o que explica a impressionante marca de 5.361 casos de leishmaniose tegumentar notificados na última década, somente na região nordeste do Estado (CosTA et al., 2005).

Considerando que os flebotomíneos são insetos de ocorrência primariamente silvestre, a mata ciliar de Urbano Santos representaria um hábitat primitivo para as espécies encontradas neste estudo. Por esse motivo estimula-se o desenvolvimento de projetos no sentido de manter a integridade desse ecossistema com o intuito de prevenir invasões de povoados rurais e até mesmo de setores periurbanos de Urbano Santos por espécies de flebotomíneos com capacidade de proliferar nesses ambientes. No Maranhão já é bem conhecida a proliferação do L. longipalpis em focos peridomiciliares e periurbanos de calazar (ARAúJo et al., 2000; CARVALHO et al., 2000), mas se essa tendência se intensificar com $L$. whitmani e outras espécies, o surgimento de focos urbanos da leishmaniose cutânea será iminente, conforme já se observa no município de Dom Pedro (LeONARDo \& REBÊLO, 2004).

Os estudos realizados no Maranhão mostraram que os flebotomíneos podem ser encontrados em atividade hematofágica em qualquer época do ano (REBÊLO, 2001; REBÊLO et al., 2001a, b). Isso ocorre porque muitas espécies se sucedem ao longo do ano, sendo poucas as que são encontradas em todos os meses. Entretanto, a tendência de maior abundância de indivíduos no período da estiagem só corrobora os achados de REBÊLo et al. (2001b) e opõe-se à maioria dos estudos realizados em outras localidades do Maranhão, onde a estação chuvosa é a de maior abundância.

Com este trabalho, ampliou-se o conhecimento sobre a estrutura de comunidade da fauna flebotomínica do Maranhão, teve-se a oportunidade de apresentar dados sobre espécies até então desconhecidas no nordeste do Estado e evidenciou-se o predomínio de L. infraspinosa e L. flaviscutellata. Neste contexto, recomenda-se estudos mais apurados no sentido de conhecer melhor as populações dessas espécies e detectar a sua importância (ou não) como vetores de Leishmania na área silvestre do município de Urbano Santos.

Agradecimentos. À Comercial e Agrícola Paineiras Ltda. por permitir a realização deste trabalho em sua propriedade. À CAPES pela concessão de bolsa de mestrado ao primeiro autor.

\section{REFERÊNCAS BIBLIOGRÁFICAS}

Araújo, J. C.; Rebêlo, J. M. M.; Carvalho, M. L. \& Barros, V. L. L. 2000. Composição dos flebotomíneos (Diptera, Psychodidae) do município da Raposa-MA, Brasil. Área endêmica de leishmanioses. Entomologia y Vectores 7:33-47.

Azevedo, A. C. R.; Rangel, E. F.; Costa, E. M.; David, J. R.; Vasconcelos A. W. \& Lopes, U. G. 1990. Natural infection of L. (Nyssomyia) whitmani (Antunes \& Coutinho,1939) by Leishmania of the braziliensis complex in Baturité, Ceará State, Northeastern Brazil. Memórias do Instituto Oswaldo Cruz 85:251.

Carvalho, M. L.; Rebêlo, J. M. M.; Araújo, J. C. \& Barros, V. L. L. 2000. Aspectos ecológicos dos flebotomíneos (Díptera, Psychodidae) do município de São José de Ribamar, MA, Brasil. Área endêmica de leishmanioses. Entomologia y Vectores 7:19-32.

Costa, J. M. L.; Rebêlo, J. M. M.; Saldanha, A. C. R.; Gama, M. E. A.; Bezerril, A. C. R. \& MaiA, A. N. S. 2005. Epidemiologia de leishmaniose tegumentar americana e perspectiva de controle 
no estado do Maranhão, Brasil. Revista do Hospital Universitário 6:32-38.

Costa, J. M. L.; Saldanha, A. C. R.; Mello e Silva, A. C.; SerraNeto, A.; Galvão, C. E. S.; Pedroso e Silva, C. M. \& Silva, A. R. 1992. Estado atual da leishmaniose cutânea difusa (LCD) no estado do Maranhão. Aspectos epidemiológicos, clínicoevolutivos. Revista da Sociedade Brasileira de Medicina Tropical 25:115-123.

Figueiredo, N. \& Andrade, G. V. 2007. Pequenos Fragmentos: Biodiversidade e Conservação. Revista Inovação 6:28-30.

IBGE (Instituto Brasileiro de Geografia e Estatística). 1984 Atlas do Maranhão. Rio de Janeiro, Edição em Convênio com o Governo do Estado do Maranhão, Instituto Brasileiro de Geografia e Estatística. 104p.

Kato, M.; Matsuda, T. \& Yamashitta, Z. 1952. Associative ecology of insects found in paddy field cultivated by various planting forms. Science Reports Tohoku University 19:291-301.

Krebs, C. J. 1989. Ecological Methodology. New York, Harper Collins. 654p.

Laroca, S. 1995. Ecologia: princípios e métodos. Petrópolis. Ed. Vozes. 197p.

Leonardo, F. S. \& RebêLo, J. M. M. 2004. A periurbanização de Lutzomyia whitmani em área de foco de leishmaniose cutânea, no Estado do Maranhão, Brasil. Revista da Sociedade Brasileira de Medicina Tropical 37:282-284.

Martin, A. M. C. B. \& RebêLo, J. M. M. 2006. Dinâmica espaçotemporal de flebotomíneos (Diptera, Psychodidae) do município de Santa Quitéria, área de cerrado do Estado do Maranhão, Brasil. Iheringia, Série Zoologia, 96:273-384.

Martins, L. M.; Rebêlo, J. M. M.; Costa, J. M. L.; Silva, A. R. \& Ferreira, L. A. 2004. Ecoepidemiologia da leishmaniose tegumentar no Município de Buriticupu, Amazônia do Maranhão, Brasil 1996 a 1998. Cadernos de Saúde Pública 20:109-118.

MayrinK, W.; Williams, P.; Coelho, M. V.; Dias, M. \& Martins, A. V. 1979. Epidemiology of dermal leishmaniasis in the Rio Doce Valley, State of Minas Gerais, Brazil. Annals of the Tropical Medicine and Parasitology 73:123-137.
Pielou, E. C. 1975. Ecological diversity. New York, John Wiley \& Sons. $165 \mathrm{p}$.

RebêLo, J. M. M. 2001. Freqüência horária e sazonalidade de Lutzomyia longipalpis (Diptera: Psychodidae: Phlebotominae) na ilha de São Luís, Maranhão, Brasil, Cadernos de Saúde Pública 17:221-227.

Rebêlo, J. M. M.; Leonardo, F. S.; Costa, J. M. L.; Pereira, Y. N. O. \& Silva, F. S. 1999. Flebotomíneos (Diptera, Psychodidae) de área endêmica de leishmanioses na região dos cerrados, estado do Maranhão, Brasil. Cadernos Saúde Pública 32:247-253.

Rebêlo J. M. M.; Mendes, W. A.; Costa, J. M. L. \& Cavaleiro, N 1996. Lista preliminar das espécies do gênero Lutzomyia Franá, 1924 (Phlebotominae, Psychodidae) do Estado do Maranhão-Brasil. Caderno Saúde Pública 12:37-45.

Rebêlo, J. M. M.; Oliveira, S. T.; Barros, V. L. L. \& Silva, F. S. 2000a. Flebotomíneos (Diptera, Psychodidae) de Lagoas, município de Buriticupu, Amazônia maranhense. I - Riqueza e abundância relativa das espécies em área de colonização recente. Revista da Sociedade Brasileira Medicina Tropical 33:11-19.

. 2000b. Flebotomíneos da Amazônia maranhense. IV - Riqueza e abundância relativa das espécies em área de colonização antiga. Entomologia y Vectores 7:61-72.

Rebêlo, J. M. M.; Oliveira, S. T.; Barros, V. L. L. \& Silva, F. S. 2001a. Flutuação sazonal dos flebotomíneos (Diptera, Psychodidae) em área de colonização recente do município de Buriticupu, Amazônia maranhense, Brasil. Revista Brasileira de Entomologia 45:11-16.

Rebêlo, J. M. M.; Oliveira, S. T.; Silva, F. S. \& Costa, J. M. L. 2001b. Sandflies (Diptera, Psychodidae) of the amazonia of Maranhão. V. Seasonal occurrence in ancient colonization area and endemic for cutaneous leishmaniasis. Revista Brasileira de Biologia 61:107-115.

Young, D. G. \& Duncan, M. A. 1994. Guide to the identification and geographic distribution of Lutzomyia sandflies in Mexico, the West Indies; Central and South America (Diptera: Psychodidae). Memories of the American Entomology Institute 54:1-881. 